\title{
A Bio-inspired knowledge system for improving combined cycle plant control tuning
}

\author{
Jose Luis Calvo-Rolle ${ }^{\mathrm{a}, *}$, Emilio Corchado ${ }^{\mathrm{b}}$ \\ a Departamento de Ingeniería Industrial, University of A Coruña, A Coruña, Spain \\ ${ }^{\mathrm{b}}$ Departamento de Informática y Automática, University of Salamanca, Salamanca, Spain
}

\section{A R T I C L E I N F O}

\section{Article history:}

Received 2 July 2012

Received in revised form

30 November 2012

Accepted 14 January 2013

Available online 26 July 2013

Keywords:

Industrial applications

Bio-inspired models

Artificial neural networks

Knowledge-based system

Auxiliary steam system control

Combined cycle plant

\begin{abstract}
A B S T R A C T
This study presents a novel bio-inspired knowledge system, based on closed loop tuning, for calculating the Proportional-Integral-Derivative (PID) controller parameters of a real combined cycle plant. The aim is to automatically achieve the best parameters according to the work point and the dynamics of the plant. To this end, several typical expressions and systems were taken into account to build the model for this multidisciplinary study. Each of these expressions is appropriated for a particular system. The novel method is empirically verified under a real case study based on an auxiliary steam system of a combined cycle plant.
\end{abstract}

(c) 2013 Elsevier B.V. All rights reserved.

\section{Introduction}

It is well known that the typical power generation plants are far from achieving an optimal operating point [40]. Consequently, these plants are not operating at an optimal point of production in economic terms. In an effort to reverse this situation a large number of Combined Cycle Power Plants have been built in recent years. These new plants allow higher efficiency levels and reduced pollutant emissions. Compared to traditional power plants, they guarantee higher efficiency and reduced emissions [41].

However, it is also well known that Combined Cycle Power Plants experience significant losses of efficiency and are usually used to control the net frequency [42]. The primary reason for this is the auxiliary services of the plant, which are not optimized [42]. Recently much research has been made in this field with the aim to improve this type of power plants and to increase their overall efficiency [41,42,49-52].

Although the PID controller is one of the most traditional control mechanisms, researchers are still working with it to improve its control action and performance [1-4]. While there are several studies oriented to the same objective, they are always tailored to

\footnotetext{
* Corresponding author. Tel.: +34981 337400; fax: +34 981337401.

E-mail addresses: jlcalvo@udc.es, jlcalvo@cdf.udc.es (J.L. Calvo-Rolle), escorchado@usal.es (E. Corchado).
}

specific systems such as, for example, Robot Fault Diagnosis [2], speed controller [3] and, mobile robot control [4].

Despite this, there are many controllers operating far below the most favorable state [7], or general system controllers that are not self-tuning. Thus it is indispensable to achieve new ways to solve this problem. In many research studies related to the PID controller, the method followed is to try to obtain the best parameters according to the system [26-30]. Another way is to achieve selftuning controller topologies [4-6].

Rule based systems are models based on the experience of human experts $[43,58]$. These experts deduce rules from a system and structure the rules according to their behavior [43]. These methods allow the implemented system to emulate the expert's behavior in a certain field $[43,44]$, and have been one of the most implemented methods in both research and operation [44]. There are several examples of those models. For instance [56] combines both rule based systems and case based reasoning to provide product design decision support. [57] shows a rule based system that complements the Risk Metrics Wealth Bench system for portfolio optimization with nonlinear cash-flows and constraints. [58] describes a robust and general rule-based approach to manage situation awareness. Bio-inspired models are inspired by nature [45], which can be applied in many systems ranging from such diverse fields as industry, network security and healthcare $[45,53,55]$. These models provide a deeper insight of biological phenomena. Bio-inspired systems provide solutions to problems that could not be solved satisfactorily by other techniques 


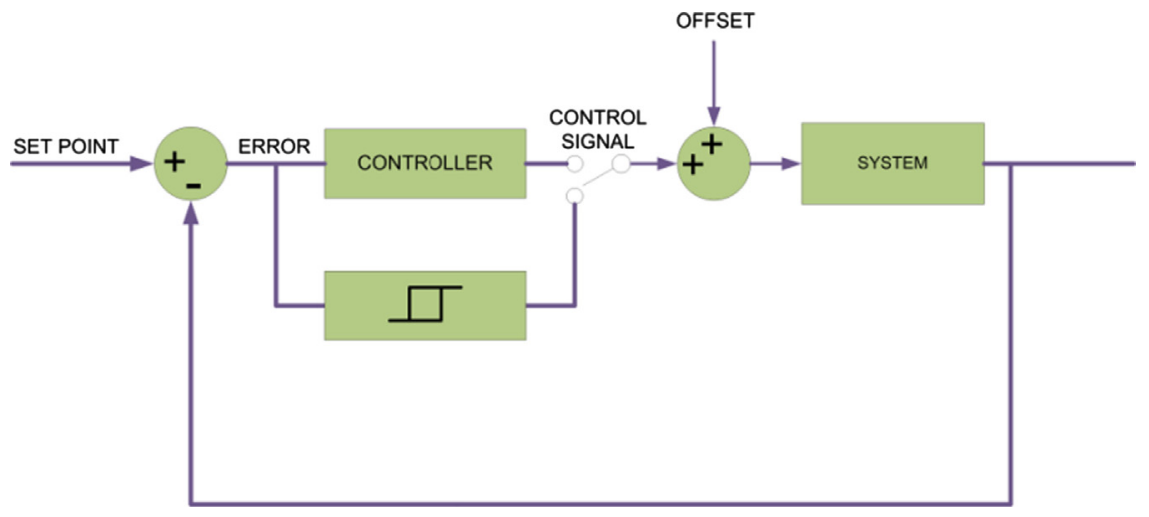

Fig. 1. Typical configuration of Relay-Feedback.

$[46,47,48]$. Some examples of Bio-inspired models are: neural networks [53], evolutionary systems [54] and the ant colony [55].

The use of rule based systems and Bio-inspired models in a knowledge system is befitting for solving the control problem of the auxiliary steam system of a combined cycle plant and improving the overall efficiency of the power plant.

The novel Bio-inspired Knowledge System (BIOKSY) presented in this study consists of two phases. The first phase is based on expert rules [31,32] and the second on a bio-inspired method [33-35]. The basic element of the model based on rules only contemplates techniques with a thoroughly tested implantation in the industry. BIOKSY is based on practical and robust methods [7,12]. There are several methods and applications that combine rule-based systems and bio-inspired models. For instance [36] shows an approach that maps a rule-based system into the neural architecture in both, the structural and the behavioral aspects. In [37] a medical application is used to test the behavior of the proposed hybrid systems. [38] studies an expert system designed to assist managers in forecasting the performance of stock prices; it was developed to demonstrate the advantages of this integrated approach and how it can enhance support for managerial decision making.

Essentially the novel method proposed in this paper achieves the best tuning parameters of a PID controller to improve a certain desired specification. Typical results of previous research [9-12] were taken into account, but it is possible to include any other method in the model.

The model is verified over the part of the cycle plant that provides electric power. While many other studies have been made to improve the operation and the efficiency of this type of industry [22-25], the objectives in this case are achieved through control optimization.

This paper is organized as follows. It begins with a brief description of the general diagram followed by an explanation of the PID controller format, and a brief review of PID controller tuning in a closed-loop. BIOKSY is then presented, followed by an empirical verification made over the auxiliary steam system of a combined cycle plant. Finally conclusions and future works are presented.

\section{PID controller}

A PID controller is a generic control loop feedback controller widely used in industrial control systems [7]. PID is the most commonly used feedback controller [8]. Essentially, a PID controller calculates an error value as the difference between the measured process variable and the desired setpoint [7]. The controller attempts to minimize the error by adjusting the process control inputs. The PID controller algorithm involves three separate constant parameters, and is accordingly sometimes called three-term control, in reference to the proportional, the integral and derivative values [10].

\subsection{PID controller format}

There are several topologies of PID controllers, but this study employs the standard format presented in Eqs. [(1),(7) and (8)].

$u(t)=K\left[e(t)+\frac{1}{T_{i}} \int_{0}^{t} e(t) d t+T_{d} \frac{d e(t)}{d t}\right]$

where ' $u$ ' is the control variable and ' $e$ ' is the control error given by ' $e=S P-y$ ' (difference between the setpoint 'SP' and conditioned output ' $y$ '). The other terms are the tuning controller parameters: proportional gain ' $K$ ', integral gain ' $T i$ ' and derivate gain ' $T d$ '.

\subsection{PID controller tuning in closed-loop}

This section presents the PID controller tuning method in closed-loop. BIOKSY is based on this tuning technique. First of all, the general procedure to calculate parameters will be presented, followed by an explanation of the procedure used to obtain the response characteristics. Finally, typical expressions used to obtain the PID controller parameters are shown.

\subsubsection{General procedure to calculate parameters}

Two steps are necessary to obtain the controller tuning parameters in closed loop:

- 1st Step. It is necessary to carry the system response to a permanent state of oscillation, after which certain characteristics of the response must be measured.

- 2nd Step. According to the information achieved from the plant response, appropriate expressions must be applied to obtain correct controller parameters for the desired specifications.

\subsubsection{Obtaining the response characteristics in closed-loop}

Different methods can be used to obtain the controller parameters. BIOKSY uses a relay-feedback method proposed by Aström and Hägglud [7]. The results are very similar to those obtained by the traditional method proposed for Ziegler-Nichols [9], but with some very important advantages including the fact that the system operates at a state that is far from unstable, and also the fact that tuning can be made at any time for any working point.

The implementation scheme of relay feedback is shown in Fig. 1. A relay with hysteresis centered on the zero value with an 
Table 1

Expressions of controller parameters.

\begin{tabular}{lllll}
\hline & $K p$ & $T i$ & $T d$ & Appl. range \\
\hline Z-N & $0.6 \times K c$ & $0.5 \times T c$ & $0.125 \times T c$ & $2<K . K c<20$ \\
Z-N Some Ov. & $0.33 \times K c$ & $T c / 2$ & $T c / 3$ & $2<K . K c<20$ \\
Z-N Whitout Ov & $0.2 \times K c$ & $T c$ & $T c / 3$ & $2<K . K c<20$ \\
Tyreus-Luyben & $0.45 \times K c$ & $2.2 \times T c$ & $T c / 6.3$ & $2<K . K c<20$ \\
\hline
\end{tabular}

amplitude $d$ and a hysteresis window of width $h$ is recommended for the general method.

The system oscillation has a period $(T c)$ with approximately the same value as the Ziegler-Nichols method. The critical gain $(K c)$ of the process is obtained by using Eq. (2), where $a$ is the peak to peak value of the oscillation.

$K_{C}=\frac{4 d}{\pi \sqrt{a^{2}-h^{2}}}$

\subsubsection{Obtaining controller parameters with formulas}

Controller parameters are obtained with the Tc and Kc characteristics of the system obtained from the previous step. Many expressions have been developed by different authors [7,9-12] with the aim of achieving items (Overshoot, peak response, settling time, rise time and so on) that improve a particular specification of the system controlled response and make the system more robust to a particular criteria (Load Disturbance or Set Point Criteria).

There are several studies with different systems which are carried out under diverse conditions where a lot of expressions were developed (to reduce noise effect, for slow systems, for systems with time delay, and so on) [39]. Additionally, control equipment manufacturers have developed their own expressions according to their product lines [39].

In this study four methods were taken into account (Table 1): Ziegler-Nichols [9-11], Ziegler-Nichols some overshoot [9], Ziegler-Nichols without overshoot [9] and Tyreus-Luyben [12]. All of them are defined for Load Disturbance rejection criteria to reduce the effect of perturbations [7].

\section{Classification techniques considered to complete the model}

This section briefly describes the three classification techniques [16-19] used to complete the novel model:

- Fisher's Linear Discriminant Analysis FLDA [14,15]. This is a technique for dimension reduction which projects the original high-dimensional data onto a low-dimensional space, where all the classes are well separated. FLDA carries out the discrimination of classes by means of hyperplanes which are derived from the training data. In the event that there are two classes, the classification of test vector $x$ is given by projecting $x$ onto the weight vector $w$ (see Eq. (3)).

$y=w^{T} x_{\text {test }}$

A projection that maximizes the class separation for two classes $C_{1}$ and $C_{2}$ is obtained to find $w$, given a training set of data vectors $x$. A good separation should be given when the projections of the class involves exposing a long distance along the direction of $w$. These projections are given by Eq. (4).

$\mu_{1}-\mu_{2}=w^{T}\left(m_{1}-m_{2}\right)$

where $\left(\mu_{1}-\mu_{2}\right)$ means the projection and $m_{i}$ represents the mean vectors of the two classes, and $w$ their projections onto $w$. The classification of a test dataset performed by FLDA provides each sample, first of all, with the projection of the sample onto $w$ and the class label. The first output corresponds to a gradual decision and the second to a binary decision. The projection is a scalar value that gives a measure of the distance in the projection between the sample and the hyperplane. This distance can be interpreted as a measure for the distinctiveness of the sample from samples of the other class. FLDA finds the best separation of two classes by maximizing the quotient of the class mean distance and the class variance. To achieve a good separation, it is desirable to have a large distance between the means.

- J48 learning algorithm [16-18]. The decision tree approach is one of the most common approaches in automatic learning and decision [16,17]. The purpose of these decision trees is to classify the data into different groups, according to the dependent variable [16]. The decision trees were obtained by using the J48 algorithm $[16,19,20]$. The J48 algorithm was chosen because of its better performance in most circumstances [20]. In general terms the decision tree classifier method involves an entropy calculation. Entropy is the probable information based on the partitioning into subcategories according to attribute. The greatest advantage is the purity of the subcategory partitions. The feature with the greatest entropy reduction is chosen as the test attribute for the present node. Let $S$ be a set made of $s$ data samples. Assume the attribute has $m$ distinct values defining $m$ distinct classes: $C_{i}$ (for $\left.i=1, \ldots, m\right)$. Let $s_{i}$ be the number of samples of $S$ in class $C_{i}$. The expected information needed to classify a given sample is given by Eq. (5).

$I\left(s_{1}, s_{2}, \ldots \ldots, s_{m}\right)=-\sum_{i=1}^{m} p_{i} \log _{2} p_{i}$

where, $p_{i}$ is the probability that $a$ random sample belongs to Class $C_{i}$ and is estimated by $s_{i} / s$. Let attribute $A$ have $v$ different values, $\left\{a_{1}, a_{2}, \ldots, a_{v}\right\}$. Attribute $A$ can be used for the partition of $S$ into $v$ subsets, $\left\{S_{1}, S_{2}, \ldots, S_{v}\right\}$, where $S_{j}$ covers those samples in $S$ that have value $a_{j}$ of $A$.

If $A$ was selected as the test attribute, then these subsets would correspond to the branches grown from the node containing the set $S$. Let $s_{i j}$ be the number of samples of class $C_{i}$ in a subset $S_{j}$. The entropy is given by Eq. (6).

$E(A)=-\sum_{j=1}^{v} I\left(s_{1 j}, s_{2 j}, \ldots \ldots, s_{m j}\right) \frac{\left(s_{1 j}, s_{2 j}, \ldots \ldots, s_{m j}\right)}{s}$

The encoding information that would be gained by branching on $A$ is presented in Eq. (7).

$\operatorname{Gain}(A)=I\left(s_{1}, s_{2}, \ldots \ldots, s_{m}\right)-E(A)$

The method uses gain ratio which applies normalization to information gain using a value defined in Eq. (8).

$\operatorname{Split}(S)=-\sum_{i=1}^{v}\left(\left|S_{i}\right| /|S|\right) \log _{2}\left(\left|S_{i}\right| / \mid S\right)$

The above value represents the information generated by splitting the training dataset $S$ into $v$ partitions corresponding to $v$ outcomes of a test on the attribute $A$.

The gain ratio is defined as:

Gain Ratio $(A)=$ Gain $(A) / \operatorname{Split}(S)$

The attribute with the highest gain ratio is selected as the splitting attribute. The non-leaf node of the decision tree generated is considered a relevant attribute.

- A multilayer perceptron (MLP) [21] is a feed forward artificial neural network $[16,18]$ characterize by its robustness and 


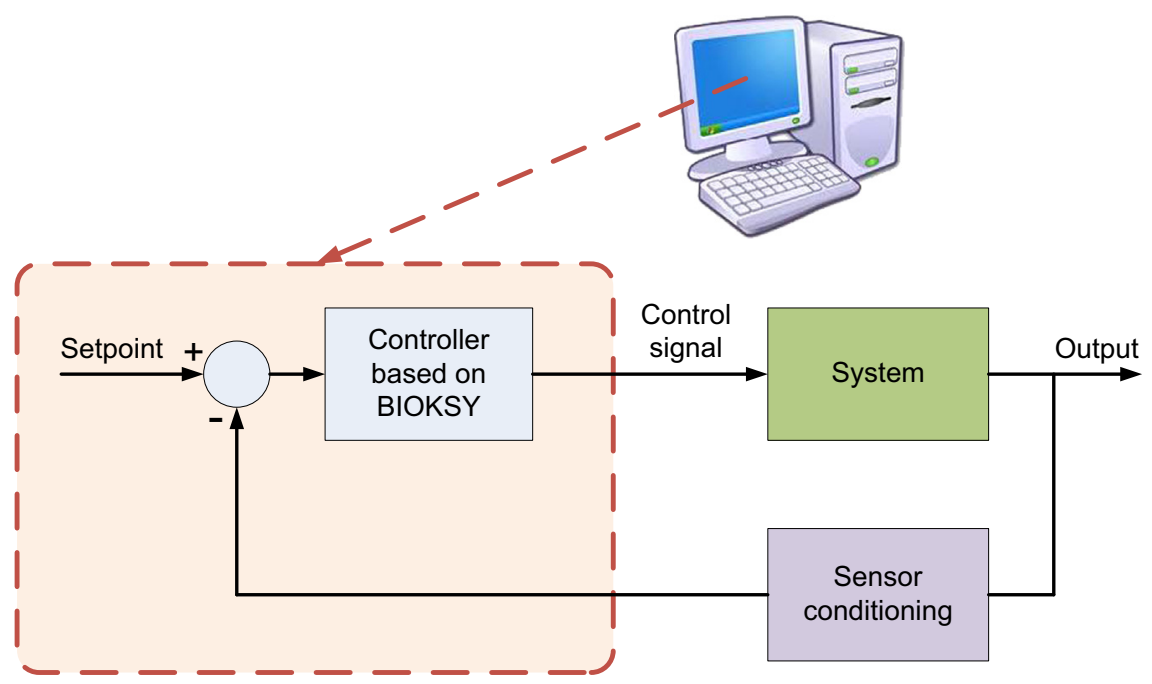

Fig. 2. System overview with a PID controller based on BIOKSY.

relatively simple structure [21]. However the ANN architecture must be carefully selected to achieve good results. Step, Linear, Log-sigmoid and Tan-sigmoid activation functions can be applied to identify the best function in each case.

\section{System overview to study the proposed bio-knowledge model}

Fig. 2 illustrates the general schema of the proposed topology where BIOKSY is tested to select the optimal controller parameters.

The novel Bio-Controller is based on the traditional PID controller in standard format, and its tuning is based on the closedloop method, specifically the relay-feedback technique. Consequently, it is more applicable in many types of cases than other methods [9]. The obtained model is shown in Fig. 3, where there is one part based on the use of rules (Part I) and another based on artificial neural networks (ANN) (Part II).

\section{BIOKSY}

BIOKSY consists of a rigorous and explicit description of the domain knowledge of the controller. It is a fusion between rules and bio-inspired techniques.

The general conceptual diagram of BIOKSY (see Fig. 4) is divided into three different blocks:

- Knowledge of existing rules. The aim of this block is to organize different rules for application ranges, authors expressions, criterions, and so on.

- Traditional knowledge link with new bio-inspired knowledge. This part is the link between the other two blocks through which information will be interpreted by the third block.

- Bio-inspired knowledge to complete the model. This block selects the most appropriate expressions to obtain the controller tuning parameters.

In general terms, BIOKSY will select the best tuning parameters, according to the system and the desired specifications of operation.

The next two subsections present the simplified bio-knowledge model. The first shows the knowledge representation corresponding

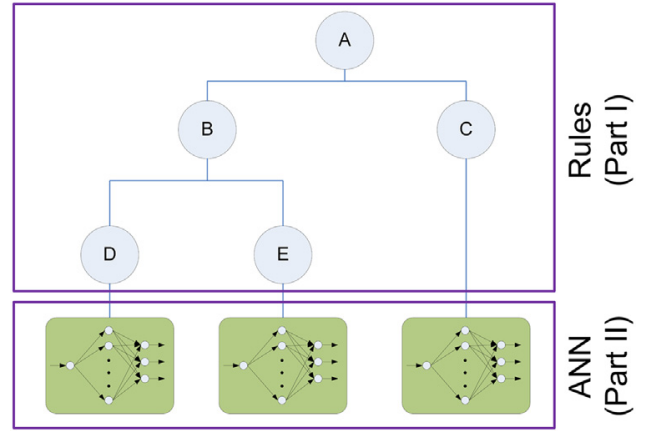

Fig. 3. Model final structure.

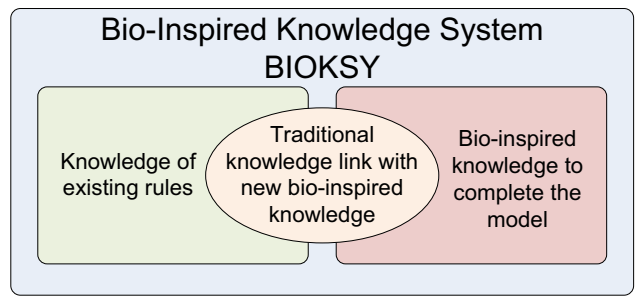

Fig. 4. Bio-Inspired Knowledge System (BIOKSY).

to the first two blocks explained before. The second gives details about the third block 'Bio-inspired knowledge to complete the model'.

\subsection{First block of the knowledge representation}

A flow chart is obtained as a result of the different methodologies of PID controller tuning in closed-loop (shown in Fig. 5). Many PID tuning rules in open loop were considered in the creation of this diagram, with the aim of achieving a generalized knowledge of the field. The following paragraphs explain the flowchart in greater details.

PID tuning in closed loop is only possible if the plant engineer can set the system operation to sustained oscillation. For this reason the flowchart starts with this premise. As explained in Section 4, there are several techniques to perform PID controller tuning in closed loop. The Relay-Feedback method is the most robust, and has achieved the best results. It is then necessary to find out if the system can achieve sustained oscillation. If it is not 


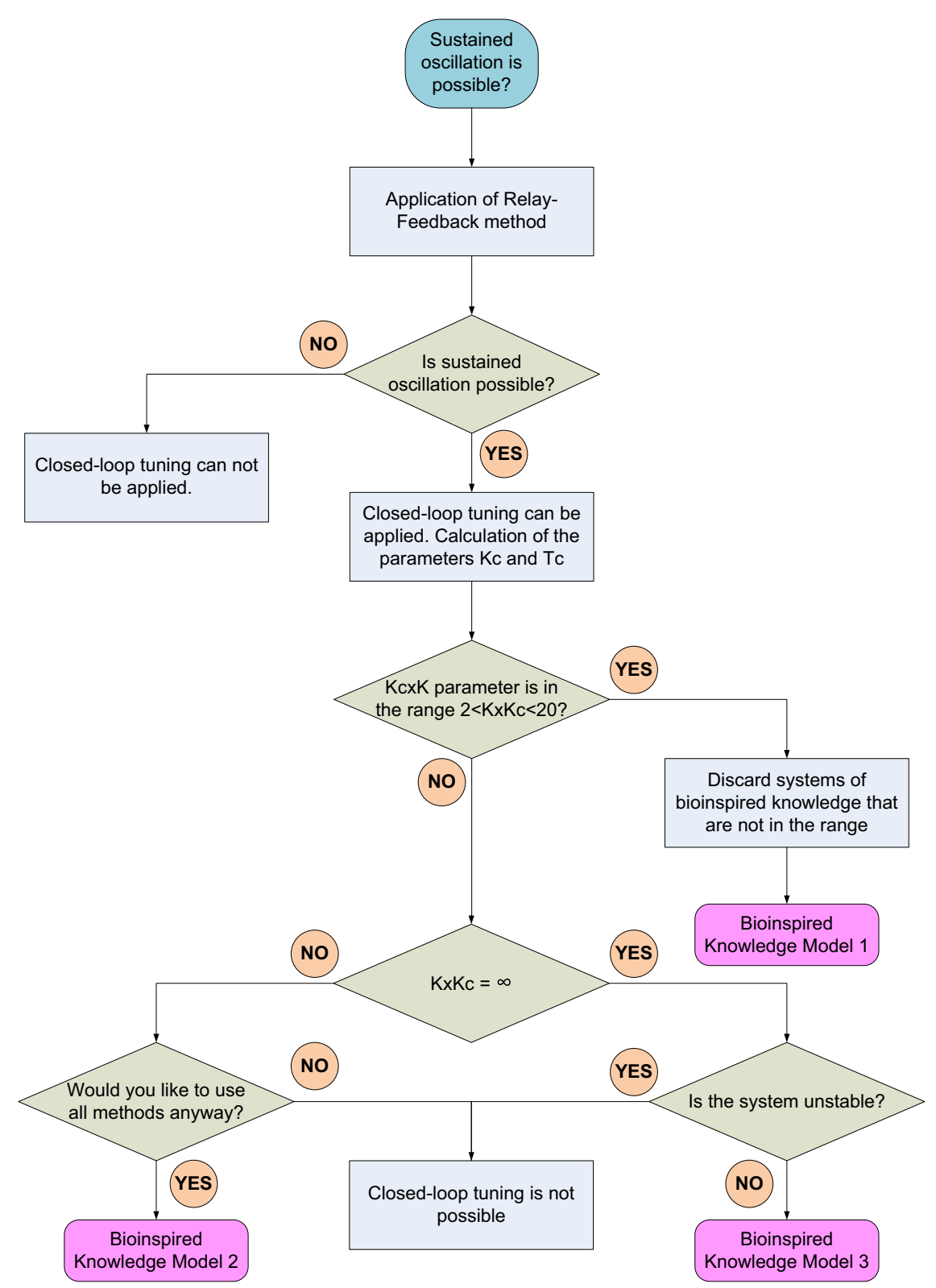

Fig. 5. Flowchart corresponding with the first part of knowledge representation.

possible, then tuning in closed loop cannot be applied. Otherwise, with the system in this state it is necessary to calculate the Tc and Kc parameters.

By knowing the Tc and Kc (see PID controller, Section 2) values it is possible to obtain the $K . K c$ indicator, where $K$ is the gain of the process. This indicator, among others, defines the best expressions to achieve the desired operation as the result of the system.

The next step in the flowchart is to determine if the K.Kc indicator value is in the range of $2<K . K c<20$. If it is within this range, then in order to fix BIOKSY all the systems that are not within this range must be discarded [7]. If these conditions are met, then it is possible to use the bio-inspired knowledge Model 1 (see Fig. 5).

If the $K . K c$ indicator is out of range, the model must be able to know if the value is infinite or not. Then it is possible to follow the flowchart in two ways:

- When K.Kc is not infinite, the operator must decide if they will nevertheless use all the expressions. If not, closed loop tuning is not applicable for the expressions being considered. Otherwise it is possible to use the bio-inspired knowledge model 2 (see Fig. 5).

- When $K . K c$ is infinite, the operator must find out if the system is unstable. If it is unstable, then closed loop tuning is not applicable. This means that the system has an integrator in its transfer function. In this case it is possible to use the bio knowledge model 3 (see Fig. 5).

\subsection{Bio-inspired knowledge to complete the model}

As shown in Fig. 5, in addition to the organization's set of rules, there are three blocks corresponding to the bio-inspired knowledge models (1, 2 and 3 ). Three techniques were applied to create these blocks: rules without applying machine learning methods, tree decision models, and artificial neural networks. The formulation of the model is described in the following paragraphs.

Model input. As seen in the flowchart (Fig. 5) and its corresponding description, the K.Kc. is a very important indicator, as it defines, for example, the application range of expressions. In all 
bio-inspired knowledge models created, K.Kc was used as the input that defines the system type. Thus, K.Kc defines the system dynamics and consequently its controllability.

Dataset for model creation. As in other studies, it is necessary to incorporate representative systems with the objective of generalizing the model as much as possible. For this reason, this study is based on the research done by Aström and Hagglund [13]. Their research includes a list of very representative systems, all of which behave similarly. Table 2 shows a list of systems included in [13] where it is possible to obtain K.Kc. In total, their study covers 32 such systems. The systems have been ordered for K.Kc value from the highest to lowest values.

As shown in Table 2, the $K . K c$ values of the first systems are very close; however the last systems have widely separated values. As a result, many systems were created to solve the problem, and in so doing, a delay time is added to the first and second order systems with Pade approximation [7]. Thus, a difference in the $K$. $K c$ values between consecutive systems of less than one is achieved. Finally there are 1704 systems that were used to obtain the model. The obtained systems are balanced by controller parameters expressions.

System specifications for each expression. Each system is tuned with the four expressions identified in Table 1. Four specifications are then tested in all cases for the step input: response time (Tr), settling time (Ts), overshoot $(O v)$ and peak time $(T p)$. As a result, it is possible to obtain the expression that gives the best specification value. Table 3 shows examples of some of the systems from Table 2 with the best specifications values for step input. The expression used to achieve this result is specified in brackets, according to the following legend: Z\&N (Ziegler-Nichols), Z\&N wOv (Ziegler-Nichols Whitout Overshoot), Z\&N sOv (ZieglerNichols some Overshoot), T\&L (Tyreus-Luyven).

Classification results: a model was created for each specification (response time, settling time, overshoot and peak time). Each of the sets of expressions is a class:

- Class A: Z-N.

- Class B: Z-N Some Ov.

- Class C: Z-N Whitout Ov

- Class D: Tyreus-Luyben

Five parameters were used to measure performance: Sensitivity (SE), Specificity (SPC), Positive Prediction Value (PPV), Negative Prediction Value (NPV) and Accuracy (ACC) (as listed in Eq. (10) to (14) respectively).

$$
\begin{aligned}
& S E=\frac{T P}{(T P+F N)} \\
& S P C=\frac{T N}{(F P+T N)} \\
& P P V=\frac{T P}{(T P+F P)} \\
& N P V=\frac{T N}{(T N+F N)} \\
& A C C=\frac{T P+T N}{(T P+T N+F P+F N)}
\end{aligned}
$$

where TP is the number of True Positive, $T N$ is the number of True Negative, FN is the number of False Negative and FP is the number of False Positive.
Table 2

\begin{tabular}{|c|c|c|}
\hline System & $\operatorname{Tr}$ & Ts \\
\hline G1 & $24.45(\mathrm{Z \& N})$ & $48.18(\mathrm{Z} \& \mathrm{~N})$ \\
\hline C6 & $0.44(\mathrm{Z} \& \mathrm{~N}$ wOv) & $48.95(\mathrm{Z} \& N)$ \\
\hline C5 & $7.92(\mathrm{Z} \& N)$ & $19.03(\mathrm{Z \& N})$ \\
\hline D2 & $0.77(\mathrm{Z} \& N)$ & $5.58(\mathrm{Z} \& \mathrm{~N})$ \\
\hline G2 & $1.05(\mathrm{Z} \& N)$ & $9.29(\mathrm{Z \& N} \mathrm{sOv})$ \\
\hline A3 & $4.01(\mathrm{Z} \& N)$ & $32.75(\mathrm{Z \& N})$ \\
\hline $\mathrm{E} 4$ & $0.85(\mathrm{Z} \& N)$ & $7.56(\mathrm{Z} \& N)$ \\
\hline B2 & $0.12(\mathrm{Z} \& N)$ & $0.9(\mathrm{~T} \& \mathrm{~L})$ \\
\hline E7 & $2.05(\mathrm{Z} \& N)$ & $19.75(\mathrm{~T} \& \mathrm{~L})$ \\
\hline B1 & $0.04(\mathrm{Z} \& N)$ & $0.33(\mathrm{~T} \& \mathrm{~L})$ \\
\hline $\mathrm{F}$ & $0.13(\mathrm{Z} \& N)$ & $1.29(\mathrm{~T} \& \mathrm{~L})$ \\
\hline System & Ov & $T p$ \\
\hline G1 & $0(\mathrm{Z \& N}$ wOv $)$ & $131.21(\mathrm{Z \& N})$ \\
\hline C6 & $0(\mathrm{Z} \& \mathrm{~N}$ wOv $)$ & $2.81(\mathrm{Z \& N}$ sOv) \\
\hline $\mathrm{C} 5$ & $0(\mathrm{Z} \& \mathrm{~N}$ wOv $)$ & $110(\mathrm{Z} \& \mathrm{~N})$ \\
\hline D2 & $0(\mathrm{Z} \& N$ wOv $)$ & $2.04(\mathrm{Z} \& N)$ \\
\hline G2 & $0(\mathrm{Z \& N}$ wOv $)$ & $4.71(\mathrm{Z} \& N)$ \\
\hline A3 & $0(\mathrm{Z \& N}$ wOv $)$ & $9.75(\mathrm{Z} \& N)$ \\
\hline E4 & $0(\mathrm{Z} \& \mathrm{~N}$ wOv $)$ & $2.25(\mathrm{Z} \& \mathrm{~N})$ \\
\hline B2 & $10.062(\mathrm{Z \& N}$ wOv) & $0.34(\mathrm{Z} \& N)$ \\
\hline E7 & $14.3172(\mathrm{Z} \& \mathrm{~N}$ wOv $)$ & $6.34(\mathrm{Z} \& N)$ \\
\hline B1 & $20.9154(\mathrm{Z} \& \mathrm{~N}$ wOv) & $0.12(\mathrm{Z} \& \mathrm{~N})$ \\
\hline $\mathrm{F}$ & 13.2937 (Z\&N wOv) & $0.36(\mathrm{Z} \& N)$ \\
\hline
\end{tabular}

Values for the $K . K c$ indicator in representative systems (G1, c6, .., F).

\begin{tabular}{rlllll}
\hline & System & \multicolumn{1}{l}{ K.Kc } & & System & K.Kc \\
\hline 1 & G1 & 0.44 & 17 & A2 & 4 \\
2 & C6 & 0.5 & 18 & B4 & 4 \\
3 & C5 & 1.1429 & 19 & C2 & 5 \\
4 & E3 & 1.5377 & 20 & G3 & 5.24 \\
5 & E2 & 1.6422 & 21 & C1 & 6.1585 \\
6 & D3 & 1.7071 & 22 & B3 & 6.7510 \\
7 & D2 & 1.7972 & 23 & A1 & 8.0011 \\
8 & G2 & 1.8812 & 24 & D6 & 8.8672 \\
9 & A3 & 1.884 & 25 & E5 & 9.7963 \\
10 & D1 & 1.9052 & 26 & G4 & 11.2414 \\
11 & E1 & 1.9052 & 27 & E6 & 16.818 \\
12 & E4 & 1.9317 & 28 & D7 & 17.5578 \\
13 & C4 & 2 & 29 & B2 & 30.2375 \\
14 & D4 & 2 & 30 & E7 & 35.1592 \\
15 & C3 & 3.2 & 31 & B1 & 110.1 \\
16 & D5 & 3.8891 & 32 & F & 167.7135 \\
\hline
\end{tabular}

Table 3

Best specifications values with tuning expressions examples.

\section{A real case study: physical description of the auxiliary steam system of a real combined cycle plant}

The aim of this research is to control the auxiliary steam of a real combined cycle plant (Fig. 6), whose goal is to supply sealed steam to both the main turbine and the degasser condenser (bubbling steam).

Fig. 6 shows the physical system where the novel method is applied to improve the control tuning. It is applied where the water supply takes place, with the fluid degassing in order to prevent an excess of non-condensable fluid within the system, which could eventually impair the functioning of the installed system. The tank is relatively small, considering the real requirements of the process. Occasionally the system becomes unstable due to its limited size. This is a very good example of where the optimal parameters for the controller would be necessary, as they would make it possible to improve the system's performance.

The general layout of the process is shown in Fig. 7. As shown, the scheme is divided into four phases. These subparts of the system will be explained on the following sections. 
Phase 1 - Supply of water into the degasser vessel. In this phase, the demineralized water goes into the system. First, the water is strained through a strainer. Control of the level in the degasser

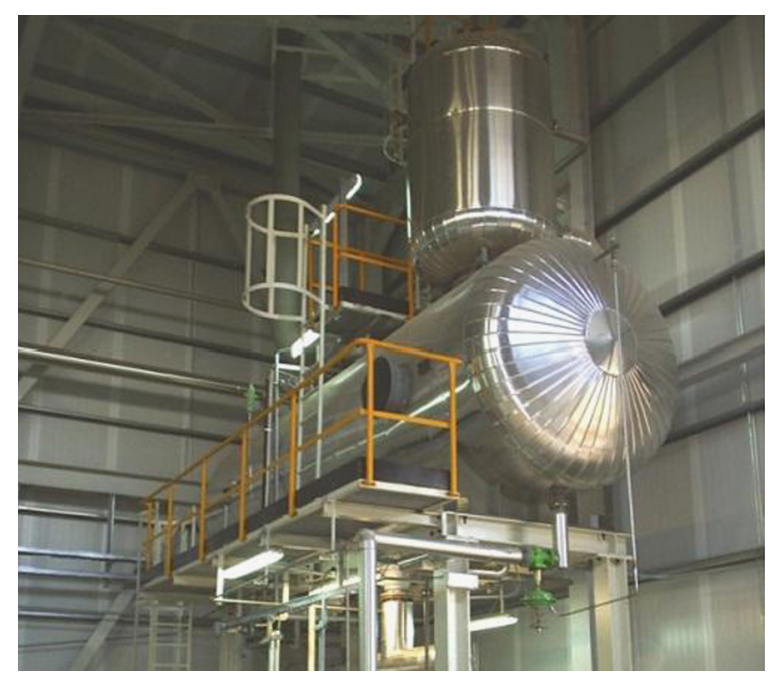

Fig. 6. General System Control Layout and physical component to which BIOKSY is applied. takes place by means of a throttle valve, which increases or decreases the flow of demineralized water.

Phase 2 - Degasser vessel. For the process at the lower side of the column a counter-current flow provides saturated steam. Saturated steam flows counter-current against the demineralized water due to the specially designed blades and diaphragms inside the degasser. This allows non-condensable particles to be removed and released out to the atmosphere at the top of the degasser. Inside the degasser, low pressure is monitored at the bottom, while pressure and heat are controlled at the top.

Phase 3 - Saturated steam supply to degasser and to demineralized water tank. Steam is conveyed to two different locations of the plant. One part of the saturated steam is piped to the water supply storage tank. The function of this tank is to heat the lowest part of the water in order to initiate degasification when starting up the plant.

The other part of the saturated steam is conveyed to the lower end of the degasser, as shown in the above sketch. The flow in this line can be controlled by a valve; usually an opening is set for the maximum flow of demineralized water allowed into the circuit.

Phase 4 - Water supply storage tank. This stage of the process corresponds to the storage of the treated water used to supply the auxiliary boiler of the combined cycle installation. In order to simplify this explanation, the process at this phase can be separated as follows:

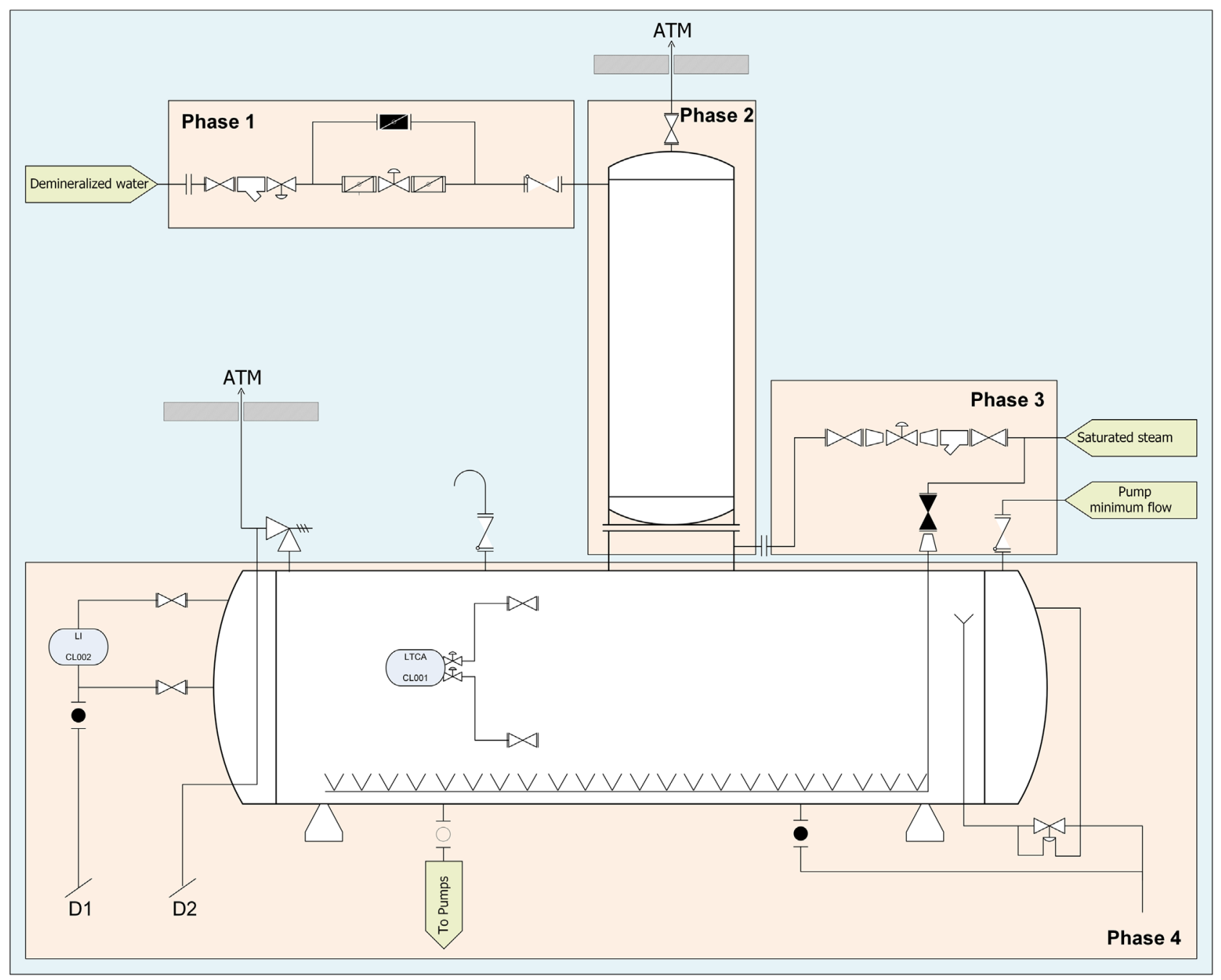

Fig. 7. General System Control Layout. 
- Intake - On top of the tank where the supply of demineralized water is located, which no longer includes incondensable particles.

- Outlet - At the bottom of the tank, treated water is pumped through a valve by the supply pumps to the auxiliary boiler in order to produce steam, which will be used later to seal the main turbine, or by the degasser to degasify.

- To ensure water level control there is a level transmitter that monitors the tank level.

- The remaining components are fundamental for the maintenance of different available sensors.

\section{Results}

\subsection{Knowledge model classificators}

This section shows the results corresponding to the classifiers created by the Bio-inspired Knowledge to complete the model explained above.

In all cases the k-fold cross-validation was used to split the dataset into a reasonable value that obtains good results. The $k$ value is 10 for all models. For the first classification method, there is a linear discriminant analysis. For the decision tree classification the algorithm chosen is J48, which contains the following configuration parameter values: the confidence threshold for pruning is 0.25 and the minimum number of instances per leaf is 2 . In the case of the MLP, tests were performed with 2 and 3 hidden layers, with best results obtained for the second value. The number of neurons in the hidden layer is within the range of 10-15. The activation functions tested in the hidden layer are linear, the log sigmoid and the tangent sigmoid (see Table 4). The best results were achieved with the tangent function, whose error rate is less than that of the other activation functions. The activation function of the output layer is the log sigmoid. The Winner Take All (WTA) technique is used to obtain the class provided by the MLP output.

Table 5 shows the percentage of correct classification using the previously mentioned techniques for the three Knowledge Models.

As can be seen in Table 5, a total of 602 systems were used for the knowledge "model 1", with an equal number of cases for each type of expression. However for the other two models (knowledge models 2 and 3) all systems were taken into account. The

Table 4

Comparision between activation function of mlp.

\begin{tabular}{|c|c|c|c|c|c|c|}
\hline \multicolumn{7}{|l|}{ Response Time (Tr) } \\
\hline \multirow[t]{2}{*}{ Model data } & \multicolumn{2}{|l|}{ Linear } & \multicolumn{2}{|l|}{ Logsig } & \multicolumn{2}{|l|}{ Tansig } \\
\hline & $\operatorname{Err}(\%)$ & No Ne. & $\operatorname{Err}(\%)$ & No Ne. & $\operatorname{Err}(\%)$ & No Ne. \\
\hline Knowledge model 1 & 38 & 11 & 31 & 12 & 4 & 13 \\
\hline Knowledge model 2 & 35 & 11 & 36 & 11 & 5 & 12 \\
\hline Knowledge model 3 & 41 & 12 & 32 & 12 & 8 & 12 \\
\hline \multicolumn{7}{|l|}{ Settling time $(T s)$} \\
\hline Knowledge model 1 & 39 & 12 & 35 & 14 & 7 & 11 \\
\hline Knowledge model 2 & 41 & 13 & 36 & 12 & 8 & 12 \\
\hline Knowledge model 3 & 40 & 14 & 38 & 13 & 8 & 12 \\
\hline \multicolumn{7}{|l|}{ Overshoot $(\mathrm{O} v)$} \\
\hline Knowledge model 1 & 35 & 12 & 33 & 13 & 6 & 14 \\
\hline Knowledge model 2 & 39 & 12 & 36 & 12 & 7 & 14 \\
\hline Knowledge model 3 & 40 & 13 & 37 & 12 & 9 & 13 \\
\hline \multicolumn{7}{|l|}{ Peak time $(T p)$} \\
\hline Knowledge model 1 & 45 & 12 & 36 & 13 & 12 & 14 \\
\hline Knowledge model 2 & 40 & 12 & 38 & 11 & 10 & 15 \\
\hline Knowledge model 3 & 42 & 12 & 38 & 13 & 11 & 14 \\
\hline
\end{tabular}

Table 5

Percentage of correct classification for three Knowledge models.

\begin{tabular}{lclll}
\hline \multirow{2}{*}{ Response time (Tr) } & & & \\
\hline Model data & Training data & \multicolumn{2}{l}{ Classification method } & \\
\cline { 3 - 5 } & & LDA & J48 & MLP \\
\hline Knowledge model 1 & 602 & 72 & 85 & $\mathbf{9 6}$ \\
Knowledge model 2 & 1786 & 74 & 87 & $\mathbf{9 5}$ \\
Knowledge model 3 & 1786 & 75 & 90 & $\mathbf{9 2}$ \\
Settling time (Ts) & & & & \\
Knowledge model 1 & 602 & 70 & 81 & $\mathbf{9 3}$ \\
Knowledge model 2 & 1786 & 69 & 83 & $\mathbf{9 2}$ \\
Knowledge model 3 & 1786 & 65 & 84 & $\mathbf{9 2}$ \\
Overshoot (Ov) & & & & \\
Knowledge model 1 & 602 & 69 & 80 & $\mathbf{9 4}$ \\
Knowledge model 2 & 1786 & 72 & 83 & $\mathbf{9 3}$ \\
Knowledge model 3 & 1786 & 72 & 81 & $\mathbf{9 1}$ \\
Peak Time (Tp) & & & & \\
Knowledge model 1 & 602 & 70 & 85 & $\mathbf{8 8}$ \\
Knowledge model 2 & 1786 & 71 & 78 & $\mathbf{9 0}$ \\
Knowledge model 3 & 1786 & 73 & 81 & $\mathbf{8 9}$ \\
\hline
\end{tabular}

Table 6

Confusion matrix for classification rate of response time of Knowledge model 2 using lda.

\begin{tabular}{lllll}
\hline Desired method & \multicolumn{4}{l}{ Method chosen by model } \\
\cline { 2 - 5 } & A & B & C & D \\
\hline A & $\mathbf{2 0 9}$ & 86 & 81 & 72 \\
B & 79 & $\mathbf{2 2 1}$ & 79 & 53 \\
C & 80 & 85 & $\mathbf{2 1 0}$ & 79 \\
D & 83 & 65 & 90 & $\mathbf{2 1 4}$ \\
TP & 209 & 221 & 210 & 214 \\
TN & 1096 & 1118 & 1082 & 1130 \\
FP & 242 & 236 & 250 & 204 \\
FN & 239 & 211 & 244 & 238 \\
SE & 0.467 & 0.512 & 0.463 & 0.473 \\
SPC & 0.819 & 0.826 & 0.812 & 0.847 \\
PPV & 0,463 & 0,484 & 0,457 & 0,512 \\
NPV & 0.821 & 0.841 & 0.816 & 0.826 \\
ACC & 0.731 & 0.750 & 0.723 & 0.753 \\
\hline
\end{tabular}

difference between the number of systems of knowledge model 1 and Knowledge models 2 and 3 is due to the fact that model 1 is created only with systems where parameter $K . K c$ is in the range of $2<K . K c<20$.

For all the cases listed in Table 5, the best configuration for each technique used was selected. To accomplish this, the confusion matrix was created in each case, comparing the desired method to the method chosen by the model. For each confusion matrix, the parameters are obtained correspond to Eqs. (10-14), which measure the classification quality. Table 5 is a summary of all classification models created where the accuracy parameter is shown. As shown in the table, the best classifications in all cases are achieved with an MLP model. The Response Time for Knowledge Model 2 is tested for the following techniques: LDA, J48 Decision Tree and MLP respectively and, is shown in Tables 6-8 as an example.

7.2. Control description and practical behavior of the proposal onto the described plant

The method was implemented in the manufacturer's software. It should be noted that this is relatively difficult because the 
software is very closed to modifications. Some tests were made, with the aim of checking the behavior of the novel model. One of the tests is described below.

As the software is very closed, the first step to obtain the response characteristics in closed-loop was performed manually. The relay feedback method, which was implemented by a human operator, centered on $800 \mathrm{~mm}$ of liquid level without a hysteresis window because the system is very slow. When the level exceeds $800 \mathrm{~mm}$ (tuning working point) the operator closes the valve (0\%), and when the level is lower than $800 \mathrm{~mm}$ the operator opens the valve (100\%). Fig. 8 shows the system when oscillation is in a

Table 7

Confusion matrix for classification rate of response time of knowledge model 2 using J48 decision tree.

\begin{tabular}{lllll}
\hline \multirow{2}{*}{ Desired method } & \multicolumn{4}{l}{ Method chosen by model } \\
\cline { 2 - 5 } & $\mathrm{A}$ & $\mathrm{B}$ & $\mathrm{C}$ & $\mathrm{D}$ \\
\hline A & $\mathbf{3 3 9}$ & 46 & 32 & 31 \\
$\mathrm{~B}$ & 21 & $\mathbf{3 4 3}$ & 40 & 28 \\
C & 38 & 44 & $\mathbf{3 1 6}$ & 56 \\
D & 49 & 45 & 50 & $\mathbf{3 0 8}$ \\
TP & 339 & 343 & 316 & 308 \\
TN & 1230 & 1219 & 1210 & 1219 \\
FP & 108 & 135 & 122 & 115 \\
FN & 109 & 89 & 138 & 144 \\
SE & & & & 0.681 \\
SPC & 0.757 & 0.794 & 0.696 & 0.914 \\
PPV & 0.919 & 0.900 & 0.908 & 0.728 \\
NPV & 0.758 & 0.718 & 0.721 & 0.894 \\
ACC & 0.919 & 0.932 & 0.898 & 0.855 \\
\hline
\end{tabular}

Table 8

Confusion matrix for classification rate of response time of knowledge model 2 using mlp.

\begin{tabular}{lllll}
\hline \multirow{2}{*}{ Desired method } & \multicolumn{4}{l}{ Method chosen by model } \\
\cline { 2 - 5 } & A & B & C & D \\
\hline A & $\mathbf{4 2 0}$ & 10 & 13 & 5 \\
B & 12 & $\mathbf{3 9 9}$ & 9 & 12 \\
C & 14 & 25 & 393 & 22 \\
D & 20 & 19 & 26 & $\mathbf{3 8 7}$ \\
TP & 420 & 399 & 393 & 387 \\
TN & 1292 & 1300 & 1284 & 1295 \\
FP & 46 & 54 & 48 & 39 \\
FN & 28 & 33 & 61 & 65 \\
SE & 0.938 & 0.924 & 0.866 & 0.856 \\
SPC & 0.966 & 0.960 & 0.964 & 0.971 \\
PPV & 0.901 & 0.881 & 0.891 & 0.908 \\
NPV & 0.979 & 0.975 & 0.955 & 0.952 \\
ACC & 0.959 & 0.951 & 0.939 & 0.942 \\
\hline
\end{tabular}

steady state ( $X$-axis shows the time variable and $Y$-axis is the level variation for the oscillation steady state). This is the appropriate time to measure $T c$ and $a$ parameters in order to calculate $K c(T c=2 \mathrm{~min}$ and $a=19 \rightarrow K c=6.7)$. Then the $K . K c$ indicator is obtained in order to apply it as an input to the model $(K . K c=0.8 \times$ $6.7=5.36)$.

The response of the system under these conditions is shown in Fig. 9. The novel proposal is able to obtain the correct expressions for the K.Kc value. With the selected expressions, the PID parameters $(K, T i$ and $\mathrm{Td})$ are calculated and are then programmed on the controller. At $K . K c=5.36$, if the operator needs to improve the overshoot, the model indicates that the operator must use the Tyreus-Luyben expressions. The PID parameter values using these expressions are: $K=3, T i=4.4$ and $T d=0.3$. The system response with a step input for the work point of the design is shown in Fig. 9. Fig. 10 shows the response with a step input for a traditional Ziegler-Nichols method in closed loop. The $X$-axis for Figs. 9 and 10 shows the time variable and the $Y$-axis shows the level of the plant tank. In both cases the dotted line shows the set point for the desired level, and the thick line shows the system response. As seen in these figures the best expressions were chosen to achieve the best overshoot between the expressions considered in the study. In both cases (Figs. 9 and 10) different set-points were programed, and as shown in each case, the selected method has a good performance.

Table 9 shows the best method selected for the model to improve the specification, and the values of the specification in each case. The first columns show the specification that the user attempts to improve. The second column shows the method chosen by the model to improve the specification of the first column. Finally the third column shows the value of the specification for the case being contemplated.

\section{Conclusions}

The novel Bio-inspired Knowledge System (BIOSKY) was presented in this study. It was successfully tested with a practical application on a real combined cycle plant with a problematic control loop where a PID controller is used. It is often the case that when no specific purpose control system is implemented, the control loop is programmed in a general form regardless of the final application. Consequently, the system provider only includes a set of parameters for the controller for the entire operating range and for different systems. For the case study in which the parameters were programmed by the provider, the plant tank level goes to oscillation state frequently. Independently of the system type, the greatest advantage offered by the method is that it ensures the most appropriate selection of the expressions needed to calculate the PID controller parameters and to improve a selected specification of the process. Good results were obtained in the performed tests, compared to the results achieved with typical expressions employed with PID controllers.

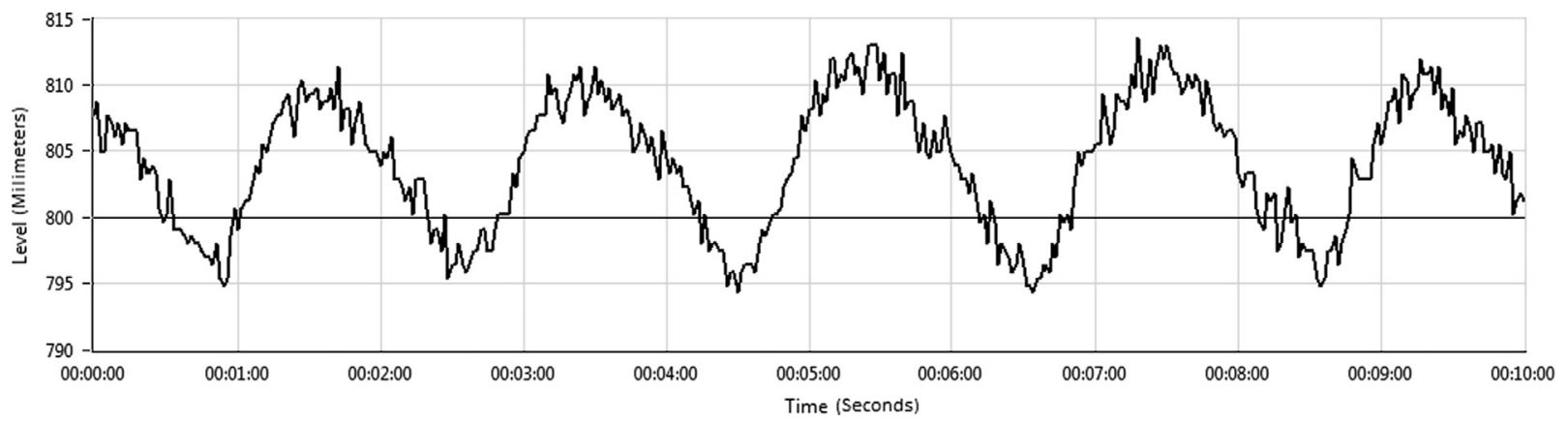

Fig. 8. System response for relay feedback centered on $800 \mathrm{~mm}$ of level. 


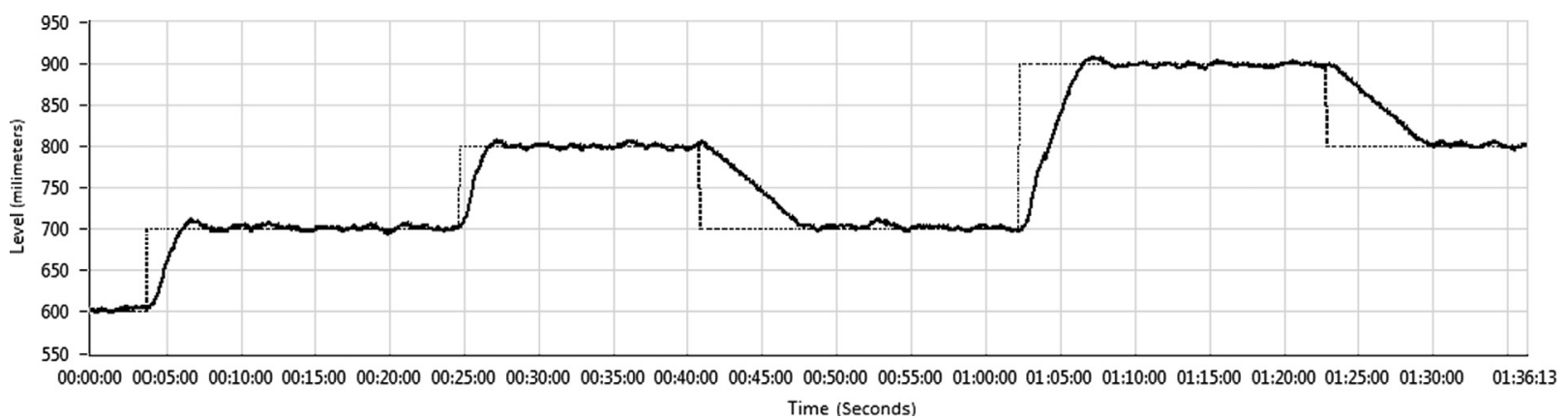

Fig. 9. System response with Tyreus-Luyben method chosen from the model.

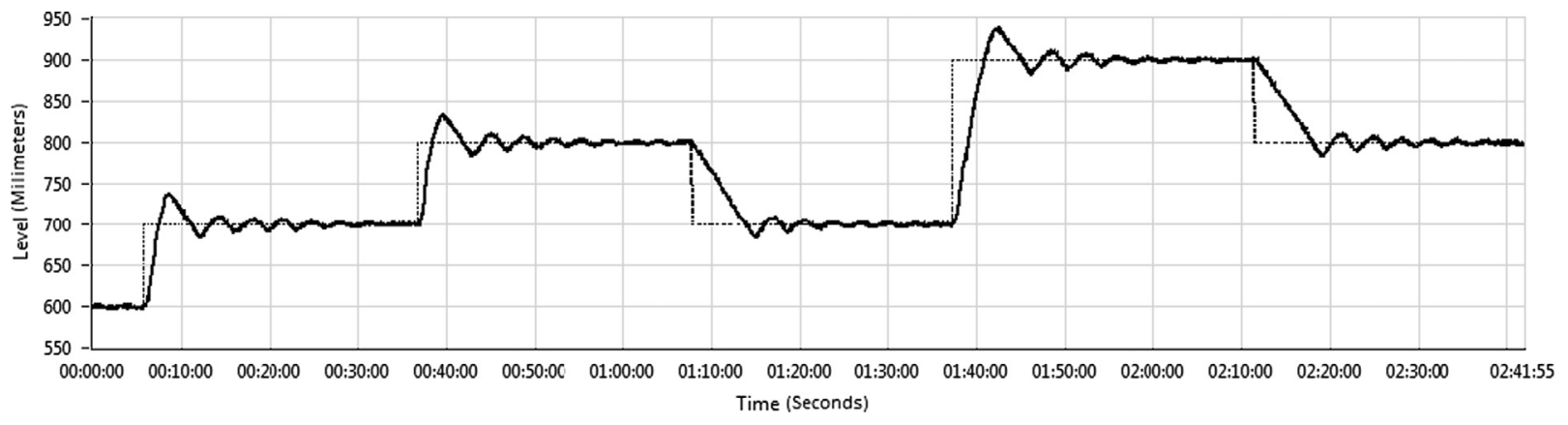

Fig. 10. System response with traditional Ziegler-Nichols method in closed-loop.

Table 9

Methods selected for the model for each specification and its values.

\begin{tabular}{lll}
\hline $\begin{array}{l}\text { Specification to } \\
\text { improve }\end{array}$ & Method chosen by model & $\begin{array}{l}\text { Specification } \\
\text { value }\end{array}$ \\
\hline $\begin{array}{ll}\text { Response time }(T r) \\
\text { Settling time }(T s)\end{array}$ & Z\&N (Ziegler-Nichols) - Class A & 1 min and 02 s \\
Overshoot $(O v)$ & T\&L (Tyreus-Luyven) - Class D & $\begin{array}{l}5 \text { min and } 56 \mathrm{~s} \\
12 \%\end{array}$ \\
Peak time $(T p)$ & Z\&N sOv(Ziegler-Nichols some & 1 min and $53 \mathrm{~s}$ \\
& Overshoot) - Class B & \\
\hline
\end{tabular}

It should be noted that the novel model is easy to expand to other system types. It is only necessary to consider the new systems that were not taken into account when creating the Bio-inspired Knowledge models. The rest of the model is completely valid.

With the aim of comparing and making the model more robust, several techniques were taken into consideration: LDA, decision trees J48 and MLP. It was shown that the MLP performed better than the other models.

Several future lines of research have been considered, two of which are of particular importance. Firstly it is necessary to consider other bio-inspired and intelligent techniques to improve the model despite the very good results that have been already obtained. Another research line will involve testing the response of nontypical industrial plants when using the new proposed system.

\section{Acknowledgment}

This research is partially supported through a project of the Spanish Ministry of Economy and Competitiveness [ref: TIN201021272-C02-01] (funded by the European Regional Development Fund) and by the Project SA405A12-2 of the JCyL. This work was also supported in the framework of the IT4Innovations Centre of Excellence project, Reg. no. CZ.1.05/1.1.00/02.0070 by operational programme Research and Development for Innovations funded by the Structural Funds of the European Union and state budget of the Czech Republic.

\section{References}

[1] C. Hsu, G. Chen, T. Lee, Robust intelligent tracking control with PID-type learning algorithm, Neurocomputing 18 (2007) 234-243.

[2] H. Liu, G. Coghill, A model-based approach to robot fault diagnosis, Knowledge-Based Systems 18 (2005) 225-233.

[3] R. Thangaraj, T.R. Chelliah, M. Pant, A. Abraham, C. Grosan, Optimal gain tuning of PI speed controller in induction motor drives using particle swarm optimization, Logic Journal of IGPL 19 (2011) 343-356, http:/dx.doi.org/ 10.1093/jigpal/jzq031.

[4] J. Ye, Adaptive control of nonlinear PID-based analog neural networks for a nonholonomic mobile robot, Neurocomputing 71 (2008) 1561-1565.

[5] J.A. Romero, R. Sanchis, P. Balaguer, PI and PID auto-tuning procedure based on simplified single parameter optimization, Journal of Process Control 21 (2011) 840-851.

[6] J. Sun, D.-hua Zhang, X. Li, J. Zhang, D.-shun Du, Smith prediction monitor AGC system based on fuzzy self-tuning PID control, Journal of Iron and Steel Research, International 17 (2010) 22-26.

[7] K.J. Astrom, T. Hagglund, Advanced PID Control, Pearson Education, Madrid 2009.

[8] Y.L. Feng, K.C. Tan, Process Identification and PID Control, John Wiley\&Sons, USA, 2009.

[9] A. Visioli, Practical PID Control, Springer-Verlag, London, 2010.

[10] M.A. Johnson, M.H. Moradi, PID Control: New identification and Design Methods, Springer-Verlag, London, 2010.

[11] H. Seki, T. Shigemasa, Retuning oscillatory PID control loops based on plant operation data, Journal of Process Control 20 (2010) 217-227.

[12] B.D. Tyreus, W.L. Luyben, Tuning PI controllers for integrator/dead time processes, Industrial Engineering Chemistry Research 31 (11) (1992) 2625-2628.

[13] K.J. Astrom, T. Hagglund, Benchmark systems for PID control preprints FAC workshop on digital control, Past present and future of PID Control Tarrasa (2000) 181-182.

[14] P.N. Belhumeur, J.P. Hespanha, D.J. Kriegman, Eigenfaces vs. Fisherfaces: recognition using class specific linear projection, IEEE Transactions on Pattern Analysis and Machine Intelligence 19 (1997) 711-720.

[15] D.L. Swets, J.J. Weng, Using discriminant eigenfeatures for image retrieval, IEEE Transactions on Pattern Analysis and Machine Intelligence 18 (8) (1996) 831-836.

[16] O. Parr, Data Mining Cookbook. Modeling Data for Marketing, Risk, and Customer Relationship Management, John Wiley \& Sons, Inc., New York, 2001.

[17] R.O. Duda, P.E. Hart, D.G. Stork, Pattern Classification, John Wiley \& Sons, Inc., Canada, 2001.

[18] T.M. Mitchell, Machine Learning, McGraw-hill, New York, 1997. 
[19] E. Frank, I. Witten, Data Mining: Practical Machine Learning Tools and Techniques, Second Edition, Morgan Kaufmann, USA, 2005.

[20] L. Rokach, O. Maimon, Data Mining with Decision Trees: Theory and Applications, World Scientific Publishing, USA, 2008.

[21] E. Alpaydin, Introduction to Machine Learning, The MIT Press, Oxford, 2009.

[22] I. Bertini, Optimizing the start-up operations of combined cycle power plants using soft computing methods, Logic Journal of IGPL 20 (2012) 648-656, http: /dx.doi.org/10.1093/jigpal/jzr013.

[23] D. Saez, F. Milla, L.S. Vargas, Fuzzy predictive supervisory control based on genetic algorithms for gas turbines of combined cycle power plants, IEEE Transactions on Energy Conversion 22 (3) (2007) 689-696.

[24] J. Álvarez López, R.N. Gómez, I.G. Moya, Practical commitment of combined cycle plants using dynamic programming, in: Proceedings of the Electric Power and Energy Conference (EPEC), IEEE, 25-27 August 2010, pp. 1-6.

[25] G. Lin, X. Junrong, D. Yiping, Modeling of combined cycle power plant based on a genetic algorithm parameter identification method, Sixth Internationa Conference on Natural Computation (ICNC), vol. 7, pp. 3369-3373, 10-12 August 2010.

[26] E.N. Sanchez, H.M. Becerra, C.M. Velez, Combining fuzzy, PID and regulation control for an autonomous mini-helicopter, Information Sciences 177 (10) (2007) 1999-2022.

[27] R.E. Precup, S. Preitl, PI-Fuzzy controllers for integral plants to ensure robust stability, Information Sciences 177 (20) (2007) 4410-4429.

[28] D.H. Kim, Hybrid GA-BF based intelligent PID controller tuning for AVR system, Applied Soft Computing 11 (1) (2011) 11-22.

[29] B. Paris, J. Eynard, S. Grieu, M. Polit, Hybrid PID-fuzzy control scheme for managing energy resources in buildings, Applied Soft Computing 11 (8) (2011) 5068-5080.

[30] K.Y. Chen, P.C. Tung, M.T. Tsai, Y.H. Fan, A self-tuning fuzzy PID-type controller design for unbalance compensation in an active magnetic bearing, Expert Systems with Applications 36 (4) (2009) 8560-8570.

[31] B. Goertzel, R. Lian, I. Arel, H. de Garis, S. Chen, A world survey of artificial brain projects, Part II: biologically inspired cognitive architectures, Neurocomputing 74 (3) (2010) 30-49.

[32] C.H. Cheng, L.Y. Wei, Y.S. Chen, Fusion ANFIS models based on multi-stock volatility causality for TAIEX forecasting, Neurocomputing 72 (18) (2009) 3462-3468

[33] K. Li, X. Hong, G.W. Irwin, Life System Modelling, Simulation, and Bio-inspired Computing (LSMS 2007), Neurocomputing 72 (3) (2008) 126-127.

[34] F. Sandoval, J. Cabestany, A. Prieto, Bio-inspired systems: computational and ambient intelligence, Neurocomputing 74 (16) (2011) 2591-2593.

[35] X. Wei, Q. Zhang, G. Cui, Some novel classification and learning methods and applications for neural networks-selected papers from the second international conference on bio-inspired computing: theories and applications, Neurocomputing 73 (4-6) (2010) 677.

[36] L.M. Fu, Integration of neural heuristics into knowledge-based inference, in: Proceedings of the International Joint Conference on Neural Networks (IJCNN) 1989, vol. 2, pp. 606.

[37] W. Ertel, C. Goller, M. Schramm, Integrating Rule Based Reasoning and Neura Networks, TU Munchen, Germany, 1995

[38] Y. Yoon, T. Guimaraes, G. Swales, Integrating artificial neural networks with rule-based expert systems, Decision Support Systems 11 (5) (1994) 497-507.

[39] A. O'Dwyer, Handbook of Pi And Pid Controller Tuning Rules, Imperial College Press, London, 2009.

[40] R. Kehlhofer, B. Rukes, F. Hannemann, F. Stirnimann., Combined-cycle gas and steam turbine power plants, PennWell Books, , 2009.

[41] W.J. Watson, The 'success' of the combined cycle gas turbine, in: Proceedings of the IEEE International Conference on Opportunities and Advances in International Electric Power Generation, 1996, p. 87-92.

[42] N. Kakimoto, K. Baba, Performance of gas turbine-based plants during frequency drops, IEEE Transactions on Power Systems 18 (3) (2003) 1110-1115.

[43] F. Hayes-Roth, et al., Building Expert Systems, Addison-Wesley, Massachusetts, Reading, MA, 1983.

[44] F. Hayes-Roth, et al., Rule-based systems, Communications of the ACM 28 (9) (1983) 921-932.

[45] A. Prieto, J. Cabestany, F. Sandoval, Computational intelligence and bioinspired systems, Neurocomputing 70 (16-18) (2007) 2701-2703.

[46] M. Sipper, Machine Nature: The Coming Age of Bio-Inspired Computing, McGraw-Hill, New York, 2002.

[47] R. Paton, Computing with Biological Metaphors, Chapman \& Hall, London, 1994.

[48] L. Nunes de Castro, J.V. Fernando, Recent Developments in Biologically Inspired Computing, IGI Global, Brazil, 2005.

[49] A.M. Bassily, Numerical cost optimization and irreversibility analysis of the triple-pressure reheat steam-air cooled GT commercial combined cycle powe plants, Applied Thermal Engineering 40 (2012) 145-160.

[50] A. Rovira, C. Sánchez, M. Muñoz, M. Valdés, M.D. Durán, Thermoeconomic optimisation of heat recovery steam generators of combined cycle gas turbine power plants considering off-design operation, Energy Conversion and Management 52 (4) (2011) 1840-1849.

[51] R. Berrios, F. Núñez, A. Cipriano, Fault tolerant measurement system based on Takagi-Sugeno fuzzy models for a gas turbine in a combined cycle power plant, Fuzzy Sets and Systems 174 (1) (2011) 114-130.

[52] C. Koch, F. Cziesla, G. Tsatsaronis, Optimization of combined cycle power plants using evolutionary algorithms, Chemical Engineering and Processing: Process Intensification 46 (11) (2007) 1151-1159.

[53] A. Herrero, E. Corchado, P. Gastaldo, R. Zunino, Neural projection techniques for the visual inspection of network traffic, Neurocomputing 72 (16-18) (2009) 3649-3658.

[54] R. Damasevicius, Complexity estimation of genetic sequences using informationtheoretic and frequency analysis methods, Informatica 21 (1) (2010) 13-30.

[55] J.C. Marinello Filho, R. Nunes de Souza, T. Abrão, Ant colony input parameters optimization for multiuser detection in DS/CDMA systems, Expert Systems with Applications 39 (17) (2012) 12876-12884.

[56] C.A. Costa, M.A. Luciano, C.P. Lima, R. Young, Assessment of a Product Range Model concept to support design reuse using rule based systems and case based reasoning, Advanced Engineering Informatics 26 (2) (2012) 292-305.

[57] YW Chen, S.H. Poon, J.B. Yang D.L. Xu, D. Zhang S. Acomb, Belief rule-based system for portfolio optimisation with nonlinear cash-flows and constraints, European Journal of Operational Research 223 (3) (2012) 775-784.

[58] M. Cimino, B. Lazzerini, F. Marcelloni, A. Ciaramella, An adaptive rule-based approach for managing situation-awareness, Expert Systems with Applications 39 (12) (2012) 10796-10811.

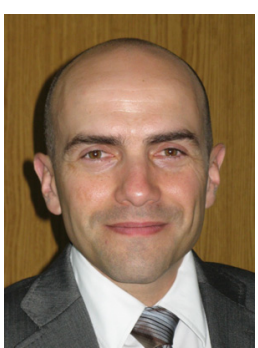

Jose Luis Calvo-Rolle was born in A Coruna, Spain, in 1974. He received the M.S. and Ph.D. degrees in Industrial Engineering from the University of Leon, Leon, Spain, in 2004, and 2007, respectively. He is Associate Professor of Automatic Control and the head of Industrial Engineering Department, Faculty of Engineering, University of A Coruna, Spain. His main research interests have been centered in applying expert system technology to the diagnosis and control systems and in intelligent training systems for control engineering, optimization and education.

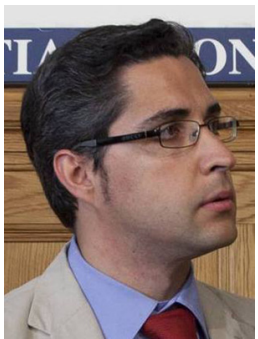

Emilio S. Corchado is an Associate Professor of Computer Science at University of Salamanca (BISITE Research Group and GICAP Research Group). He is chair of the IEEE Systems, Man and Cybernetics-Spanish Chapter; member of the IEEE Spanish Section Board and member of the IEEE R8 PA subcommittee. He is or was a member of some European networks of Excellence as Nature-inspired Smart Information Systems NiSIS) and COST Action IC0806: Intelligent Monitoring, Control and Security of Critical Infrastructure Systems (IntelliCIS). He is reviewer and evaluator for the European Commission for the 7FP, since 2007.

His research interests include neural networks, with a particular focus on exploratory projection pursuit, maximum likelihood hebbian learning, self-organising maps, multiple classifier systems and Hybrid Artificial Systems and its applications to industrial and environmental problems. He has published over 170 peer-reviewed articles in a range of topics from modelling of industrial processes, knowledge management and risk analysis, intrusion detection systems, food industry, artificial vision, and so on.

$\mathrm{He}$ is co-editor in Chief of the International Journal of Computational Intelligence Research (IJCIR) and member of the Editorial Boards of the International Journal of Computational Intelligence and Applications (IJCIA) and also of the International Journal of Reasoning-based Intelligent Systems (IJRIS). Dr. Corchado is a guest editor of several special issues of reputed journals as Information Science (Elsevier), Knowledge and Information Systems (Springer), Neurocomputing (Elsevier), International Journal of Neural Systems (World Scientific), Logic Journal Of the IGPL (Oxford Journals), Neural Networks World (Institute of Computer Science AS CR and Faculty of Transportation Sciences), Expert Systems (Wiley-Blackwell). He is general chair for the following conferences: International Conference on Hybrid Artificial Intelligence Systems (HAIS), International Conference on Soft Computing Models in Industrial and Environmental Applications (SOCO) and International Conference on Computational Intelligence in Security for Information Systems (CISIS). 